\title{
Vitali Lemma approach to differentiation on a time scale
}

\author{
by \\ Chuan Jen Chyan (Taipei) and Andrzej Fryszkowski (Warszawa)
}

\begin{abstract}
A new approach to differentiation on a time scale $\mathbb{T}$ is presented. We give a suitable generalization of the Vitali Lemma and apply it to prove that every increasing function $f: \mathbb{T} \rightarrow \mathbb{R}$ has a right derivative $f_{+}^{\prime}(x)$ for $\mu_{\Delta}$-almost all $x \in \mathbb{T}$. Moreover, $\int_{[a, b)} f_{+}^{\prime}(x) d \mu_{\Delta} \leq f(b)-f(a)$.
\end{abstract}

The theory of time scales has received much attention since Hilger's [4] initial paper introduced the unifying theory for continuous and discrete calculus. Subsequent major development of the calculus on time scales is due to Agarwal and Bohner [1], Bohner and Peterson [2, 3], and Kaymakcalan et al. [5].

In this paper, we are concerned with the covering theorem of Vitali on time scales. It is well known that the Vitali Lemma is the basic tool for the development of differentiation theory for Riemann and Lebesgue integrals. A similar treatment of differentiation theory on time scales is given.

Before introducing the problems of interest for this paper, we present some definitions and notations which are common to the recent literature.

Definition 1. A nonempty closed subset, $\mathbb{T}$, of $\mathbb{R}$, endowed the subspace topology, is called a time scale. For $t<\sup \mathbb{T}$ and $r\rangle \inf \mathbb{T}$, define the forward jump operator, $\sigma$, and the backward jump operator, $\varrho$, by

$$
\sigma(t)=\inf \{\tau \in \mathbb{T}: \tau>t\} \in \mathbb{T}, \quad \varrho(r)=\sup \{\tau \in \mathbb{T}: \tau<r\} \in \mathbb{T} .
$$

If $\sigma(t)>t$, then $t$ is said to be right-scattered, and if $\varrho(r)<r$, then $r$ is said to be left-scattered. If $\sigma(t)=t$ and $t<\sup \mathbb{T}$, then $t$ is said to be right-dense, and if $\varrho(r)=r$ and $r>\inf \mathbb{T}$ then $r$ is said to be left-dense.

The sets of right-dense and left-dense points will be denoted by $D_{R}$ and $D_{L}$ respectively.

2000 Mathematics Subject Classification: Primary 26A24, 28A15; Secondary 46G05, 39A05, $28 \mathrm{~A} 05$.

Key words and phrases: Vitali Lemma, time scale, differentiation, increasing function. 
Definition 2. For $x: \mathbb{T} \rightarrow \mathbb{R}$ and $t \in \mathbb{T}$ (if $t=\sup \mathbb{T}$, assume $t$ is not left-scattered), define the delta derivative of $x(t), x^{\Delta}(t)$, to be the number (when it exists) with the property that, for any $\varepsilon>0$, there is a neighborhood, $U$, of $t$ such that

$$
\left|[x(\sigma(t))-x(s)]-x^{\Delta}(t)[\sigma(t)-s]\right| \leq \varepsilon|\sigma(t)-s|
$$

for all $s \in U$. If $F^{\Delta}(t)=h(t)$, then define the integral by

$$
\int_{a}^{t} h(s) \Delta s=F(t)-F(a) .
$$

Definition 3. For $a, b \in \mathbb{T}$, define the closed interval $[a, b]$ in $\mathbb{T}$ by

$$
[a, b]=\{t \in \mathbb{T}: a \leq t \leq b\} .
$$

Open intervals, half-open intervals etc. are defined similarly. For an arbitrary interval $P \subset \mathbb{T}$ we denote its length by

$$
l(P)=b-a .
$$

Consider the family $\mathcal{F}_{R}=\{[a, b): a, b \in \mathbb{T}\}$ and for an arbitrary set $A \subset \mathbb{T}$ put

$$
m^{*}(A)=\inf \left\{\sum_{i=1}^{\infty} l\left(P_{i}\right): P_{i} \in \mathcal{F}_{R} \text { and } A \subset \bigcup_{i=1}^{\infty} P_{i}\right\} .
$$

One can notice that $m^{*}$ is an outer measure on $2^{\mathbb{T}}$. By the Carathéodory Extension Theorem, $m^{*}$ defines a $\sigma$-field $\mathcal{L}=\mathcal{L}_{\Delta}$ of measurable sets $A \in \mathcal{L}$ satisfying the condition

$$
m^{*}(E)=m^{*}(E \cap A)+m^{*}\left(E \cap A^{\prime}\right)
$$

for every $E \subset \mathbb{T}$, where $A^{\prime}=\mathbb{T} \backslash A$. Define $\mu_{\Delta}=\left.m^{*}\right|_{\mathcal{L}}$. Following Bohner $\&$ Peterson [2] the measure $\mu_{\Delta}$ is called the $\Delta$-measure, while $\mathcal{L}$ is the family of $\Delta$-measurable sets. Note that any $P \in \mathcal{F}_{R}$ is $\Delta$-measurable with $\mu_{\Delta}(P)=l(P)$ and any compact set $K \subset \mathbb{T} \backslash\{\max \mathbb{T}\}$ is of finite $\Delta$-measure. Moreover, any Borel set is $\Delta$-measurable.

Similarly, consider the family $\mathcal{F}_{L}=\{(a, b]: a, b \in \mathbb{T}\}$ and define

$$
m_{*}(A)=\inf \left\{\sum_{i=1}^{\infty} l\left(P_{i}\right): P_{i} \in \mathcal{F}_{L} \text { and } A \subset \bigcup_{i=1}^{\infty} P_{i}\right\} .
$$

Now the Carathéodory procedure leads to the $\nabla$-measure $\mu_{\nabla}$ and $\nabla$-measurable sets $\mathcal{L}_{\nabla}$.

As usual we say that a certain property is satisfied $\Delta$-almost everywhere $(\Delta$-a.e.) or for $\Delta$-almost all ( $\Delta$-a.a. $)$ points if the set of points where the property does not hold has $\Delta$-measure zero. Similarly we define the notions of $\nabla$-almost everywhere ( $\nabla$-a.e.) and $\nabla$-almost all ( $\nabla$-a.a.). 
Notice that

$$
m_{*}(-A)=m^{*}(A), \quad m^{*}(-A)=m_{*}(A),
$$

where the outer measures on the right-hand sides are taken on the time scale $\mathbb{T}$, while on the left on $-\mathbb{T}$.

Our goal in this paper is to give an extension of the formula (1) to the Lebesgue integrals

$$
\int_{[a, b)} f_{+}^{\prime}(t) d \mu_{\Delta} \leq f(b)-f(a), \quad \int_{(a, b]} f_{-}^{\prime}(t) d \mu_{\nabla} \leq f(b)-f(a),
$$

where the derivatives $f_{+}^{\prime}(t)$ and $f_{-}^{\prime}(t)$ are understood a.e. This requires a new approach to differentiation on the time scale $\mathbb{T}$ by the use of a suitable version of the Vitali Lemma.

Before we formulate and prove it we need the following properties of the outer measures $m_{*}$ and $m^{*}$.

Proposition 1. (i) For any $A \subset D_{R}$ we have $m^{*}(A) \leq m_{*}(A)$.

(ii) For any $A \subset D_{L}$ we have $m_{*}(A) \leq m^{*}(A)$.

(iii) For any $A \subset D_{L} \cap D_{R}$ we have $m_{*}(A)=m^{*}(A)$.

Proof. We shall show just (i), since (ii) follows from (i), and (iii) is a simple consequence of the previous ones.

Fix $\varepsilon>0$ and take $R_{j}=\left(a_{j}, b_{j}\right]$ such that

$$
A \subset \bigcup_{j=1}^{\infty} R_{j}, \quad \sum_{j=1}^{\infty} l\left(R_{j}\right) \leq m_{*}(A)+\varepsilon .
$$

Set $N_{1}=\left\{j: b_{j}\right.$ is right-scattered $\}$ and $N_{2}=\left\{j: b_{j}\right.$ is right-dense $\}$. For $j \in N_{1}$ take $P_{j}=\left[a_{j}, b_{j}\right)$, while for $j \in N_{2}$ choose $P_{j}=\left[a_{j}, c_{j}\right)$, where $c_{j}>b_{j}$ is a point in $\mathbb{T}$ such that $c_{j}-b_{j}<\varepsilon / 2^{j}$. Observe that

$$
A \subset \bigcup_{j=1}^{\infty} P_{j}, \quad \sum_{j=1}^{\infty} l\left(P_{j}\right) \leq \sum_{j=1}^{\infty} l\left(R_{j}\right)+\varepsilon \leq m_{*}(A)+2 \varepsilon .
$$

Thus $m^{*}(A) \leq m_{*}(A)+2 \varepsilon$ and since $\varepsilon>0$ is arbitrary we have $m^{*}(A) \leq$ $m_{*}(A)$, which ends the proof.

Proposition 2. For a sequence of intervals $\left[a_{i}, b_{i}\right)$ with $a_{i} \in D_{R}, i=$ $1,2, \ldots$, and any $A \subset T$, we have

$$
m^{*}\left(A \cap \bigcup_{i=1}^{\infty}\left[a_{i}, b_{i}\right)\right)=m^{*}\left(A \cap \bigcup_{i=1}^{\infty}\left(a_{i}, b_{i}\right)\right) .
$$

Similarly for intervals $\left(a_{i}, b_{i}\right]$ with $b_{i} \in D_{L}, i=1,2, \ldots$, we have

$$
m_{*}\left(A \cap \bigcup_{i=1}^{\infty}\left(a_{i}, b_{i}\right]\right)=m_{*}\left(A \cap \bigcup_{i=1}^{\infty}\left(a_{i}, b_{i}\right)\right) \text {. }
$$


Proof. By (4) it is enough to show (5). Notice that always

$$
m^{*}\left(A \cap \bigcup_{i=1}^{\infty}\left[a_{i}, b_{i}\right)\right) \geq m^{*}\left(A \cap \bigcup_{i=1}^{\infty}\left(a_{i}, b_{i}\right)\right) .
$$

The equality is obvious if $m^{*}\left(A \cap \bigcup_{i=1}^{\infty}\left(a_{i}, b_{i}\right)\right)=\infty$.

If $m^{*}\left(A \cap \bigcup_{i=1}^{\infty}\left(a_{i}, b_{i}\right)\right)<\infty$ then for every $\varepsilon>0$ there exist $R_{j} \in \mathcal{F}_{R}$, $j=1,2, \ldots$, such that

$$
A \cap \bigcup_{i=1}^{\infty}\left(a_{i}, b_{i}\right) \subset \bigcup_{j=1}^{\infty} R_{j}
$$

and

$$
\sum_{j=1}^{\infty} l\left(R_{j}\right)<m^{*}\left(A \cap \bigcup_{i=1}^{\infty}\left(a_{i}, b_{i}\right)\right)+\varepsilon .
$$

Choose $P_{i}=\left[a_{i}, c_{i}\right) \in \mathcal{F}_{R}, i=1,2, \ldots$, such that $l\left(P_{i}\right)<\varepsilon / 2^{i}$. Then

$$
A \cap \bigcup_{i=1}^{\infty}\left[a_{i}, b_{i}\right) \subset \bigcup_{j=1}^{\infty} R_{j} \cup \bigcup_{i=1}^{\infty} P_{i}
$$

and hence

$$
m^{*}\left(A \cap \bigcup_{i=1}^{\infty}\left[a_{i}, b_{i}\right)\right) \leq \sum_{j=1}^{\infty} l\left(R_{j}\right)+\sum_{i=1}^{\infty} l\left(P_{i}\right)<m^{*}\left(A \cap \bigcup_{i=1}^{\infty}\left(a_{i}, b_{i}\right)\right)+2 \varepsilon .
$$

Now letting $\varepsilon \rightarrow 0$ we obtain (5).

Consider the families $\mathcal{F}_{0 R}=\left\{P \in \mathcal{F}_{R}: l(P)>0\right\}$ and $\mathcal{F}_{0 L}=\left\{P \in \mathcal{F}_{L}\right.$ : $l(P)>0\}$. Observe that $\mathcal{F}_{0 R}$ covers $D_{R}$ in the sense of Vitali, while $\mathcal{F}_{0 L}$ covers $D_{L}$. Indeed, if $x \in D_{R}$ then there is a decreasing sequence $\left\{t_{n}\right\} \subset \mathbb{T}$ such that $\lim t_{n}=x$ and hence the intervals $\left[x, t_{n}\right) \in \mathcal{F}_{0 R}$ satisfy $\lim l\left(\left[x, t_{n}\right)\right)$ $=0$. The case of $\mathcal{F}_{0 L}$ is similar.

We shall show that the following analogues of the Vitali Lemma hold:

Lemma 1. (a) (Vitali Lemma for $\Delta$-measure). Let $E \subset D_{R}$ be a set with $m^{*}(E)<\infty$. Then for every $\varepsilon>0$ there exist pairwise disjoint intervals $P_{1}, \ldots, P_{N} \in \mathcal{F}_{0 R}$ such that

$$
m^{*}\left(E \backslash \bigcup_{n=1}^{N} P_{n}\right)<\varepsilon
$$

(b) (Vitali Lemma for $\nabla$-measure). Let $E \subset D_{L}$ be a set with $m_{*}(E)$ $<\infty$. Then for every $\varepsilon>0$ there exist pairwise disjoint intervals $P_{1}, \ldots, P_{N}$ $\in \mathcal{F}_{0 L}$ such that

$$
m_{*}\left(E \backslash \bigcup_{n=1}^{N} P_{n}\right)<\varepsilon .
$$


Proof. We discuss only case (a) because the arguments used can easily be adapted to (b). Since $m^{*}(E)<\infty$, there exists $W=\bigcup_{i=1}^{\infty} R_{i} \supset E$ with $R_{i} \in$ $\mathcal{F}_{0 R}$ and $\sum_{i=1}^{\infty} l\left(R_{i}\right)<\infty$. Therefore $\mu_{\Delta}(W)=m^{*}(W) \leq \sum_{i=1}^{\infty} l\left(R_{i}\right)<\infty$. Set $\mathcal{F}_{W}=\left\{P \in \mathcal{F}_{0 R}: P \subset W\right\}$ and observe that $\mathcal{F}_{W}$ still covers $E$ in the sense of Vitali. We choose by induction a family $\left\{P_{n}\right\} \subset \mathcal{F}_{W}$ of pairwise disjoint intervals as follows: Let $P_{1} \in \mathcal{F}_{W}$ be any interval and assume that $P_{1}, \ldots, P_{n} \in \mathcal{F}_{W}$ have already been chosen.

If $E \subset \bigcup_{i=1}^{n} P_{i}$ we are done. If $E \backslash \bigcup_{i=1}^{n} P_{i} \neq \emptyset$ then for any $x \in E \backslash \bigcup_{i=1}^{n} P_{i}$ there exists an interval $P \in \mathcal{F}_{W}$ such that

$$
P \cap \bigcup_{i=1}^{n} P_{i}=\emptyset
$$

Thus the number

$$
k_{n}=\sup \left\{l(P): P \cap \bigcup_{i=1}^{n} P_{i}=\emptyset, P \in \mathcal{F}_{W}\right\}
$$

satisfies

$$
0<k_{n} \leq m^{*}(W)<\infty .
$$

Therefore one can find $P_{n+1} \in \mathcal{F}_{W}$ such that

$$
P_{n+1} \cap \bigcup_{i=1}^{n} P_{i}=\emptyset, \quad \frac{1}{2} k_{n}<l\left(P_{n+1}\right) \leq k_{n} .
$$

Notice that

$$
\frac{1}{2} \sum_{n=1}^{\infty} k_{n} \leq \sum_{n=1}^{\infty} \mu_{\Delta}\left(P_{n}\right)=\mu_{\Delta} \bigcup_{n=1}^{\infty} P_{n} \leq \mu_{\Delta}(W)<\infty .
$$

Hence $k_{n} \rightarrow 0$ as well as $l\left(P_{n}\right) \rightarrow 0$. Let $P_{n}=\left[a_{n}, b_{n}\right)$. Define

$$
\begin{aligned}
& c_{n}=\inf \left\{t \in \mathbb{T}: t \geq 3 a_{n}-2 b_{n}\right\}, \\
& d_{n}=\sup \left\{t \in \mathbb{T}: t \leq 3 b_{n}-2 a_{n}\right\},
\end{aligned}
$$

and observe that $c_{n}, d_{n} \in \mathbb{T}$ with $c_{n} \geq a_{n}$ and $b_{n} \leq d_{n}$. Thus

$$
J_{n}=\left[c_{n}, d_{n}\right) \supset P_{n}, \quad l\left(J_{n}\right) \leq\left(3 b_{n}-2 a_{n}\right)-\left(3 a_{n}-2 b_{n}\right)=5 l\left(P_{n}\right) .
$$

Now fix $\varepsilon>0$ and take $N$ such that

$$
\sum_{n=N+1}^{\infty} l\left(P_{n}\right)<\frac{\varepsilon}{5}
$$

Hence

$$
\sum_{n=N+1}^{\infty} l\left(J_{n}\right)<\varepsilon
$$


Let

$$
X=E \backslash \bigcup_{n=1}^{N} P_{n} .
$$

To establish the lemma we need to show that

$$
m^{*}(X)<\varepsilon .
$$

Take an arbitrary point $x \in X$. Since $x \notin \bigcup_{i=1}^{N} P_{i}$ we can find $P \in \mathcal{F}_{W}$ with $x \in P$ and

$$
P \cap \bigcup_{i=1}^{N} P_{i}=\emptyset .
$$

Notice that

$$
P \cap \bigcup_{i=1}^{\infty} P_{i} \neq \emptyset .
$$

Indeed, otherwise $P \cap \bigcup_{i=1}^{n} P_{i}=\emptyset$ for every $n=1,2, \ldots$ and therefore $0<l(P) \leq k_{n}$, which is a contradiction, since $k_{n} \rightarrow 0$. From (12) and (13) we conclude that

$$
P \cap \bigcup_{i=N+1}^{\infty} P_{i} \neq \emptyset .
$$

Thus there is $i \geq N+1$ such that

$$
P \cap P_{i} \neq \emptyset \text {. }
$$

Take $n$ to be the smallest possible integer $i$ exceeding $N+1$ and satisfying (14). One can easily notice that

$$
P \cap \bigcup_{i=1}^{n-1} P_{i}=\emptyset .
$$

Hence $0<l(P) \leq k_{n-1}<2 l\left(P_{n}\right)$ and if $P=[a, b)$ we have

$$
b-a<2\left(b_{n}-a_{n}\right) \text {. }
$$

We claim that $x \in J_{n}$. To see this take $z \in P \cap P_{n}$ and observe that

$$
\left|x-\frac{a_{n}+b_{n}}{2}\right| \leq|x-z|+\left|z-\frac{a_{n}+b_{n}}{2}\right|<(b-a)+\frac{b_{n}-a_{n}}{2}<\frac{5\left(b_{n}-a_{n}\right)}{2} \text {. }
$$

Thus $3 a_{n}-2 b_{n}<x<3 b_{n}-2 a_{n}$ and therefore $c_{n} \leq x \leq d_{n}$. To deduce the claim it remains to show that $x \neq d_{n}$. Assume to the contrary that $x=d_{n}$. Then

$$
b>x=d_{n} \geq b_{n} .
$$

Hence $b>3 b_{n}-2 a_{n}$, since if $b \leq 3 b_{n}-2 a_{n}$, then $b \leq d_{n}$. We also have $b_{n}>a$, since $a \leq z<b_{n}$. So finally

$$
b-a>\left(3 b_{n}-2 a_{n}\right)-b_{n}=2\left(b_{n}-a_{n}\right),
$$


which contradicts (15). Therefore $x \in J_{n} \subset \bigcup_{i=N+1}^{\infty} J_{i}$. But $x \in X$ was arbitrarily chosen, so

$$
X \subset \bigcup_{i=N+1}^{\infty} J_{i}
$$

and hence by (10) we have

$$
m^{*}(X) \leq \sum_{n=N+1}^{\infty} l\left(J_{n}\right)=\sum_{n=N+1}^{\infty} \mu_{\Delta}\left(J_{n}\right)<\varepsilon
$$

which completes the proof.

Usually the Vitali Lemma is applied to problems concerning differentiation. The same occurs for the time scale $\mathbb{T}$, but in order to talk about derivatives of a function $f: \mathbb{T} \rightarrow \mathbb{R}$ we have to distinguish between right-dense, left-dense, right-scattered and left-scattered points.

Let $x \in D_{R}$ be a right-dense point. Then the quantities

$$
D^{+} f(x)=\limsup _{t \rightarrow x^{+}} \frac{f(t)-f(x)}{t-x}, \quad D_{+} f(x)=\liminf _{t \rightarrow x^{+}} \frac{f(t)-f(x)}{t-x}
$$

will be called, respectively, the right upper and right lower derivatives of $f$ at $x$.

If $x$ is right-scattered then we put

$$
D^{+} f(x)=D_{+} f(x)=\frac{f(\sigma(x))-f(x)}{\sigma(x)-x} .
$$

Similarly, for $x \in D_{L}$ being a left-dense point we define

$$
D^{-} f(x)=\limsup _{t \rightarrow x^{-}} \frac{f(t)-f(x)}{t-x}, \quad D_{-} f(x)=\liminf _{t \rightarrow x^{-}} \frac{f(t)-f(x)}{t-x},
$$

and if $x$ is left-scattered then we put

$$
D^{-} f(x)=D_{-} f(x)=\frac{f(\varrho(x))-f(x)}{\varrho(x)-x} .
$$

One can easily observe that $D^{+} f(x) \geq D_{+} f(x)$ and $D^{-} f(x) \geq D_{-} f(x)$.

We say that $f$ is right differentiable at $x$ if $D^{+} f(x)=D_{+} f(x) \neq \pm \infty$. In this case that common value is called the right derivative of $f$ at $x$ and denoted by $f_{+}^{\prime}(x)$. Analogously, $f_{-}^{\prime}(x)=D^{-} f(x)=D_{-} f(x) \neq \pm \infty$ is called the left derivative of $f$ at $x$.

Finally, $f$ is differentiable at $x$ if $f_{+}^{\prime}(x)=f_{-}^{\prime}(x)$, and that common value is denoted by $f^{\prime}(x)$ and called the derivative of $f$ at $x$.

The derivatives just introduced have similar properties to the usual ones. We just need to notice that for $g(x)=-f(-x)$ defined on the time scale $-\mathbb{T}$ we have

$$
D^{-} g(x)=-D_{+} f(-x), \quad D_{-} g(x)=-D^{+} f(-x),
$$


and

$$
g_{+}^{\prime}(x)=-f_{-}^{\prime}(-x), \quad g_{+}^{\prime}(x)=-f_{-}^{\prime}(-x) .
$$

In what follows we shall develop a theory of differentiation distinguishing right and left derivatives. We begin with the following observation:

TheOREM 1. Let $f: \mathbb{T} \rightarrow \mathbb{R}$ be an increasing function. Then:

(i) $f$ is right differentiable $\Delta$-a.e. on $D_{R}$;

(ii) $f$ is left differentiable $\nabla$-a.e. on $D_{L}$;

(iii) $f$ is differentiable $\Delta$-a.e. and $\nabla$-a.e. on $D_{L} \cap D_{R}$.

The derivatives $f_{+}^{\prime}$ and $f_{-}^{\prime}$ are measurable, nonnegative and for every $a, b \in \mathbb{T} \backslash\{\inf \mathbb{T}$, sup $\mathbb{T}\}$ we have

$$
\int_{[a, b)} f_{+}^{\prime}(t) d \mu_{\Delta} \leq f(b)-f(a), \quad \int_{(a, b]} f_{-}^{\prime}(t) d \mu_{\nabla} \leq f(b)-f(a) .
$$

Proof. We prove just (iii), since (i) is similar, while (ii) can be deduced from (i) for $g(x)=-f(-x)$ defined on $-\mathbb{T}$. We need to show that the set of points where both right and left derivatives are unequal has both $\Delta$ and $\nabla$ measures zero, i.e.

$$
m^{*}(E)=m_{*}(E)=0 .
$$

We may consider only the set

$$
E=\left\{x \in D_{R} \cap D_{L}: f_{+}^{\prime}(x)>f_{-}^{\prime}(x)\right\}
$$

since the set arising from the opposite inequality can be similarly handled. Observe that $E$ can be represented as the union of the sets

$$
E_{n, \alpha, \beta}=\left\{x \in D_{R} \cap D_{L} \cap\left(m_{n}, M_{n}\right): f_{+}^{\prime}(x)>\alpha>\beta>f_{-}^{\prime}(x)\right\} .
$$

for all rationals $\alpha>\beta>0$ and $n=1,2, \ldots$, where

$$
m_{n}=\inf \{t \in \mathbb{T}: t \geq-n\}, \quad M_{n}=\sup \{t \in \mathbb{T}: t<n\} .
$$

Moreover from Proposition 1 we know that $m^{*}\left(E_{n, \alpha, \beta}\right)=m_{*}\left(E_{n, \alpha, \beta}\right)=: s$. Hence it is sufficient to check that

$$
s=0 .
$$

Pick an arbitrary $\varepsilon>0$ and choose $W=\bigcup_{i=1}^{\infty} R_{i} \supset E_{n, \alpha, \beta}$ with $R_{i} \in$ $\mathcal{F}_{0 L}$ and $\sum_{i=1}^{\infty} l\left(R_{i}\right)<s+\varepsilon$. Observe that for each $x \in E_{n, \alpha, \beta}$ there is an arbitrarily small interval $P=(t, x] \subset W$ such that

$$
f(x)-f(t)<\beta(x-t)=\beta l(P) .
$$

Thus the family

$$
\mathcal{F}_{W}=\left\{P=(a, b] \in \mathcal{F}_{0 L}: P \subset W, b \in D_{L}, f(b)-f(a)<\beta l(P)\right\}
$$


covers $E_{n, \alpha, \beta}$ in the sense of Vitali. Hence by Lemma 1, we can choose a finite collection $\left\{P_{1}, \ldots, P_{N}\right\} \subset \mathcal{F}_{W}$ of pairwise disjoint intervals such that

$$
m_{*}\left(E_{n, \alpha, \beta} \backslash \bigcup_{i=1}^{N} P_{i}\right)<\varepsilon .
$$

Let $P_{i}=\left(a_{i}, b_{i}\right]$ and set $A=E_{n, \alpha, \beta} \cap \bigcup_{i=1}^{N}\left(a_{i}, b_{i}\right) \subset D_{L} \cap D_{R}$. Then by Proposition 1 and (6) we have

$$
m^{*}(A)=m_{*}(A)>s-\varepsilon .
$$

By the construction for each $i \in\{1, \ldots, N\}$ we have

$$
f\left(b_{i}\right)-f\left(a_{i}\right)<\beta\left(b_{i}-a_{i}\right)=\beta l\left(P_{i}\right) .
$$

Summing up these inequalities we obtain

$$
\sum_{i=1}^{N}\left(f\left(b_{i}\right)-f\left(a_{i}\right)\right)<\sum_{i=1}^{N} \beta l\left(P_{i}\right)<\beta(s+\varepsilon) .
$$

Now each $z \in A \subset E_{n, \alpha, \beta}$ admits an arbitrarily small interval $I=[z, s) \subset$ $\left(a_{i}, b_{i}\right)$, for some $i \in\{1, \ldots, N\}$, such that

$$
f(s)-f(z)>\alpha(s-z)=\alpha l(I) .
$$

Therefore the family

$$
\begin{array}{r}
\mathcal{F}_{1}=\left\{I=[a, b) \in \mathcal{F}_{W}: I \subset\left(a_{i}, b_{i}\right) \text { for some } i \in\{1, \ldots, N\}\right. \\
\text { and } f(b)-f(a)>\alpha l(I)\}
\end{array}
$$

covers $A$ in the sense of Vitali. Now applying Lemma 1 again we can choose a finite collection $\left\{I_{1}, \ldots, I_{M}\right\} \subset \mathcal{F}_{1}$ of pairwise disjoint intervals such that

$$
m^{*}\left(A \backslash \bigcup_{k=1}^{M} I_{k}\right)<\varepsilon
$$

Let $I_{k}=\left[c_{k}, d_{k}\right)$ and set $B=A \cap \bigcup_{k=1}^{M} I_{k}$. Then

$$
m^{*}(B)>m^{*}(A)-\varepsilon>s-2 \varepsilon,
$$

and for each $k \in\{1, \ldots, M\}$ we have

$$
f\left(d_{k}\right)-f\left(c_{k}\right)>\alpha\left(d_{k}-c_{k}\right)=\alpha l\left(I_{k}\right) .
$$

Hence

$$
\sum_{k=1}^{M}\left(f\left(d_{k}\right)-f\left(c_{k}\right)\right)>\alpha \sum_{k=1}^{M} l\left(I_{k}\right)>\alpha m^{*}(B)>\alpha(s-2 \varepsilon) .
$$

But each interval $I_{k}$ is contained in some interval $P_{i}$. For given $i$, set $N(i)=$ $\left\{k: I_{k} \subset P_{i}\right\}=\left\{k_{1}, \ldots, k_{m}\right\}$, where the numbering is such that

$$
a_{i} \leq c_{k_{1}}<d_{k_{1}} \leq c_{k_{2}}<d_{k_{2}} \leq \ldots \leq c_{k_{m}}<d_{k_{m}} \leq b_{i} .
$$


Thus we obtain

$$
\begin{aligned}
\sum_{k \in N(i)}( & \left.f\left(d_{k}\right)-f\left(c_{k}\right)\right) \\
& =f\left(d_{k_{m}}\right)-f\left(c_{k_{1}}\right)+\sum_{r=1}^{m-1}\left(f\left(c_{k_{r+1}}\right)-f\left(d_{k_{r}}\right)\right) \leq f\left(b_{i}\right)-f\left(a_{i}\right),
\end{aligned}
$$

since $f$ is increasing. Hence

$$
\sum_{k=1}^{M}\left(f\left(d_{k}\right)-f\left(c_{k}\right)\right)=\sum_{i=1}^{N} \sum_{k \in N(i)}\left(f\left(d_{k}\right)-f\left(c_{k}\right)\right) \leq \sum_{i=1}^{N}\left(f\left(b_{i}\right)-f\left(a_{i}\right)\right)
$$

and so

$$
\alpha(s-2 \varepsilon)<\beta(s+\varepsilon) .
$$

Recall that $\varepsilon>0$ was arbitrarily chosen, so we have $\beta s \geq \alpha s$. But $\beta<\alpha$, hence $s$ has to be 0 .

To show (17) it is enough to prove that

$$
\int_{[a, b)} f_{+}^{\prime}(x) d \mu_{\Delta} \leq f(b)-f(a)
$$

since the other relation follows from (16) upon replacing $f(t)$ by $h(t)=$ $-f(-t)$. Observe that (i)-(iii) give the existence of sets $D_{R}^{0} \subset D_{R}$ and $D^{0} \subset D_{R} \cap D_{L}$ with

$$
m^{*}\left(D_{R} \backslash D_{R}^{0}\right)=0, \quad m^{*}\left(D_{R} \cap D_{L} \backslash D^{0}\right)=0
$$

and such that the limits

$$
\lim _{t \rightarrow x^{+}} \frac{f(t)-f(x)}{t-x} \text { and } \lim _{t \rightarrow x} \frac{f(t)-f(x)}{t-x}
$$

exist (finite or not), respectively, for $x \in D_{R}^{0}$ and for $x \in D^{0}$ (for rightscattered points by definition). Define

$$
D=\left(D_{R} \backslash D_{R}^{0}\right) \cup\left[\left(D_{R} \cap D_{L}\right) \backslash D^{0}\right]^{\prime}=D_{R}^{\prime} \cup\left[D_{R}^{0} \backslash D_{L}\right] \cup D_{R}^{0} \cap D_{L}
$$

and observe that by (21),

$$
m^{*}\left(D^{\prime}\right)=0
$$

Consider

$$
g(x)= \begin{cases}\lim _{t \rightarrow x^{+}} \frac{f(t)-f(x)}{t-x} & \text { for } x \in D_{R}^{0} \\ +\infty & \text { for } x \in D_{R} \backslash D_{R}^{0} \\ \frac{f(\sigma(x))-f(x)}{\sigma(x)-x} & \text { for } x \in T \backslash D_{R} .\end{cases}
$$

Observe that $f$ is right differentiable whenever $g(x)$ is finite for $x \in D$, and then $g(x)=f_{+}^{\prime}(x)$. We shall construct a sequence of nonnegative simple functions $g_{n}:[a, b) \rightarrow \mathbb{R}$ with the following properties: 
(a) $g_{n}(x)$ tends to $g(x)$ for $x \in[a, b) \cap D$;

(b) $\int_{[a, b)} g_{n}(x) d \mu_{\Delta} \leq f(b)-f(a)$.

The construction goes as follows. Take $\delta=(b-a) / n$ and consider a partition $\mathcal{P}_{n}$ of $[a, b)$ given by $a=t_{0}<t_{1}<\ldots<t_{N}=b$ such that for each $i \in\{1, \ldots, N\}$ either

$$
t_{i}-t_{i-1} \leq \delta
$$

or

$$
t_{i}-t_{i-1}>\delta, \quad \varrho\left(t_{i-1}\right)=t_{i}
$$

Such a partition $\mathcal{P}_{n}$ exists by Lemma 5.7 in Bohner \& Peterson [3]. Define

$$
g_{n}(x)=\frac{f\left(t_{i}\right)-f\left(t_{i-1}\right)}{t_{i}-t_{i-1}} \quad \text { for } x \in\left[t_{i-1}, t_{i}\right) .
$$

We shall show that $g_{n}(x)$ tends to $g(x)$ for $x \in D$. Denote by $\left[t_{i-1}^{n}(x), t_{i}^{n}(x)\right)$ $\in \mathcal{P}_{n}$ the subinterval containing $x$. We have to consider the following cases:

1. $x \in \mathbb{T} \backslash D_{R}$ ( $x$ is right-scattered). Then for sufficiently large $n$ we have $\sigma(x)-x>\delta=(b-a) / n$. Therefore $x$ and $\sigma(x)$ have to be consecutive division points of $\mathcal{P}_{n}$. But then

$$
g_{n}(x)=\frac{f(\sigma(x))-f(x)}{\sigma(x)-x}=g(x) .
$$

2. $x \in D_{R}^{0} \backslash D_{L}$ ( $x$ is left-scattered and right-dense). Then for sufficiently large $n$ we have $x-\varrho(x)>\delta=(b-a) / n$ and therefore $t_{i-1}^{n}(x)=x$ with $t_{i}^{n}(x) \rightarrow x^{+}$. But then

$$
g_{n}(x)=\frac{f\left(t_{i}^{n}\right)-f(x)}{t_{i}^{n}-x} \rightarrow g(x)
$$

3. $x \in D_{R}^{0} \cap D_{L}(x$ is left- and right-dense). Then

$$
\lim _{t \rightarrow x} \frac{f(t)-f(x)}{t-x}=g(x)
$$

and for each $n$ we have $t_{i}^{n}(x)-t_{i-1}^{n}(x)<\delta=(b-a) / n$. Hence $t_{i-1}^{n}(x) \rightarrow x^{-}$ and $t_{i}^{n}(x) \rightarrow x^{+}$. But then

$$
\frac{f\left(t_{i-1}^{n}(x)\right)-f(x)}{t_{i-1}^{n}(x)-x} \rightarrow g(x), \quad \frac{f\left(t_{i}^{n}(x)\right)-f(x)}{t_{i}^{n}(x)-x} \rightarrow g(x)
$$

We claim that

$$
\frac{f\left[t_{i}^{n}(x)\right]-f\left[t_{i-1}^{n}(x)\right]}{t_{i}^{n}(x)-t_{i-1}^{n}(x)} \rightarrow g(x)
$$

Indeed: 
(a) If $g(x)$ is finite, then for every $\varepsilon>0$ and sufficiently large $n$ we have

$$
\begin{gathered}
{[g(x)-\varepsilon]\left[x-t_{i-1}^{n}(x)\right] \leq f(x)-f\left[t_{i-1}^{n}(x)\right] \leq[g(x)+\varepsilon]\left[x-t_{i-1}^{n}(x)\right],} \\
{[g(x)-\varepsilon]\left[t_{i}^{n}(x)-x\right] \leq f\left[t_{i}^{n}(x)\right]-f(x) \leq[g(x)+\varepsilon]\left[t_{i}^{n}(x)-x\right] .}
\end{gathered}
$$

Adding those inequalities we obtain

$$
\begin{aligned}
{[g(x)-\varepsilon]\left[t_{i}^{n}(x)-t_{i-1}^{n}(x)\right] } & \leq f\left[t_{i}^{n}(x)\right]-f\left[t_{i-1}^{n}(x)\right] \\
& \leq[g(x)+\varepsilon]\left[t_{i}^{n}(x)-t_{i-1}^{n}(x)\right]
\end{aligned}
$$

or equivalently

$$
g(x)-\varepsilon \leq \frac{f\left[t_{i}^{n}(x)\right]-f\left[t_{i-1}^{n}(x)\right]}{t_{i}^{n}(x)-t_{i-1}^{n}(x)} \leq g(x)+\varepsilon .
$$

Thus

$$
g(x)-\varepsilon \leq g_{n}(x) \leq g(x)+\varepsilon
$$

and the latter shows that $g_{n}(x) \rightarrow g(x)$.

(b) Suppose $g(x)$ is infinite, say $+\infty$ (for $-\infty$ we proceed similarly). Then for any $K>0$ and sufficiently large $n$ we have

$$
\begin{aligned}
K\left[x-t_{i-1}^{n}(x)\right] & \leq f(x)-f\left[t_{i-1}^{n}(x)\right], \\
K\left[t_{i}^{n}(x)-x\right] & \leq f\left[t_{i}^{n}(x)\right]-f(x) .
\end{aligned}
$$

Adding again we obtain

$$
K \leq \frac{f\left[t_{i}^{n}(x)\right]-f\left[t_{i-1}^{n}(x)\right]}{t_{i}^{n}(x)-t_{i-1}^{n}(x)} .
$$

Thus

$$
\liminf _{n \rightarrow \infty} \frac{f\left[t_{i}^{n}(x)\right]-f\left[t_{i-1}^{n}(x)\right]}{t_{i}^{n}(x)-t_{i-1}^{n}(x)} \geq K,
$$

and this shows that also in this case $g_{n}(x) \rightarrow g(x)$.

From the Fatou Lemma we now conclude that

$$
\int_{[a, b)} g(t) d \mu_{\Delta} \leq \liminf _{n \rightarrow \infty} \int_{[a, b)} g_{n}(t) d \mu_{\Delta} .
$$

But

$$
\begin{aligned}
\int_{[a, b)} g_{n}(t) d \mu_{\Delta} & =\sum_{i=1}^{N} \int_{\left[t_{i-1}, t_{i}\right)} \frac{f\left(t_{i}\right)-f\left(t_{i-1}\right)}{t_{i}-t_{i-1}} d \mu_{\Delta} \\
& =\sum_{i=1}^{N}\left[f\left(t_{i}\right)-f\left(t_{i-1}\right)\right]=f(b)-f(a)
\end{aligned}
$$

and therefore

$$
\int_{[a, b)} g(t) d \mu_{\Delta} \leq f(b)-f(a)
$$


Since $g(t) \geq 0$ for $\Delta$-a.a. points in $[a, b)$, the latter shows that $g$ is $\Delta$ integrable and hence finite $\Delta$-a.e. in $[a, b)$. Thus $f$ is right differentiable a.e. in $[a, b)$ and $g=f_{+}^{\prime}$ a.e. in $[a, b)$, which completes the proof.

\section{References}

[1] R. P. Agarwal and M. Bohner, Basic calculus on time scales and some of its applications, Results Math. 35 (1999), 3-22.

[2] M. Bohner and A. Peterson, Dynamic Equations on Time Scales, Birkhäuser, Boston, 2001.

[3] - - -, Advances in Dynamic Equations on Time Scales, Birkhäuser, Boston, 2003.

[4] S. Hilger, Analysis on a measure chain. A unified approach to continuous and discrete calculus, Results Math. 18 (1990), 18-56.

[5] B. Kaymakcalan, V. Lakshmikantham and S. Sivasundaram, Dynamic Systems on Measure Chains, Kluwer, Boston, 1996.

[6] H. L. Royden, Real Analysis, Macmillan, 1988.

Department of Mathematics

Tamkang University

Taipei 251, Taiwan

E-mail: chuanjen@mail.tku.edu.tw

URL: http://www.tku.edu.tw
Faculty of Mathematics and Information Science Warsaw University of Technology Plac Politechniki 1 00-661 Warszawa, Poland E-mail: fryszko@alpha.mini.pw.edu.pl URL: http://www.alpha.mini.pw.edu.pl 